\title{
In-frame deletion in the seventh immunoglobulin- like repeat of filamin $C$ in a family with myofibrillar myopathy
}

Alexey Shatunov ${ }^{1}$, Montse Olivé ${ }^{2}$, Zagaa Odgerel ${ }^{1}$, Christine Stadelmann-Nessler ${ }^{3}$, Kerstin Irlbacher ${ }^{4}$, Frank van Landeghem ${ }^{4,13}$, Munkhuu Bayarsaikhan ${ }^{5}$, Hee-Suk Lee ${ }^{1}$, Bertrand Goudeau ${ }^{6}$, Patrick F Chinnery ${ }^{7}$, Volker Straub ${ }^{7}$, David Hilton-Jones ${ }^{8}$, Maxwell S Damian ${ }^{9}$, Anna Kaminska ${ }^{10}$, Patrick Vicart ${ }^{6}$, Kate Bushby ${ }^{7}$, Marinos C Dalakas ${ }^{11}$, Nyamkhishig Sambuughin ${ }^{5}$, Isidro Ferrer ${ }^{2}$, Hans H Goebel ${ }^{12}$ and Lev G Goldfarb*,

\footnotetext{
${ }^{1}$ National Institute of Neurological Disorders and Stroke, National Institutes of Health, Bethesda, MD, USA; ${ }^{2}$ Institut de Neuropatologia, Ciutat Sanitària i Universitària de Bellvitge, Hospitalet de Llobregat, Barcelona, Spain;

${ }^{3}$ Universitätsmedizin der Georg-August-Universität Göttingen, Göttingen, Germany; ${ }^{4}$ Charité-Universitätsmedizin Berlin, Berlin, Germany; ${ }^{5}$ Uniformed Services University of the Health Sciences, Bethesda, MD, USA; ${ }^{6}$ UFR de Biochimie, Université Paris, Denis Diderot, Paris, France; ${ }^{7}$ Institute of Human Genetics, Newcastle upon Tyne, UK; ${ }^{8}$ John Radcliffe Hospital, Oxford, UK; ${ }^{9}$ University Hospitals of Leicester, Leicester, UK; ${ }^{10}$ Medical University of Warsaw, Warsaw, Poland; ${ }^{11}$ Thomas Jefferson University, Philadelphia, PA, USA; ${ }^{12}$ Mainz University Medical Center, Mainz, Germany
}

Myofibrillar myopathies (MFMs) are an expanding and increasingly recognized group of neuromuscular disorders caused by mutations in DES, CRYAB, MYOT, and ZASP. The latest gene to be associated with MFM was FLNC; a p.W2710X mutation in the 24th immunoglobulin-like repeat of filamin C was shown to be the cause of a distinct type of MFM in several German families. We studied an International cohort of 46 patients from 39 families with clinically and myopathologically confirmed MFM, in which DES, CRYAB, $M Y O T$, and ZASP mutations have been excluded. In patients from an unrelated family a 12-nucleotide deletion (c.2997_3008del) in FLNC resulting in a predicted in-frame four-residue deletion (p.Val930_Thr933del) in the seventh repeat of filamin C was identified. Both affected family members, mother and daughter, but not unrelated control individuals, carried the p.Val930_Thr933del mutation. The mutation is transcribed and, based on myopathological features and immunoblot analysis, it leads to an accumulation of dysfunctional filamin $C$ in the myocytes. The study results suggest that the novel p.Val930_Thr933del mutation in filamin C is the cause of MFM but also indicate that filamin C mutations are a comparatively rare cause of MFM.

European Journal of Human Genetics (2009) 17, 656-663; doi:10.1038/ejhg.2008.226; published online 3 December 2008

Keywords: myofibrillar myopathy; desmin-related myopathy; limb-girdle muscular dystrophy; filamin C; small deletion mutation; German family

\footnotetext{
*Correspondence: Dr LG Goldfarb, National Institute of Neurological Disorders and Stroke, National Institutes of Health, 5625 Fishers Lane, Room 4S06, Bethesda, MD 20892-9404, USA. Tel: + 1301402 1480; Fax: + 1301496 6341; E-mail: GoldfarbL@ninds.nih.gov

${ }^{13}$ Current address: Institute of Neuropathology, University of Bonn Medical Centre, Bonn 53105, Germany

Received 11 June 2008; revised 20 October 2008; accepted 28 October 2008; published online 3 December 2008
}

Introduction

Myofibrillar myopathy (MFM) is a general term identifying a group of heterogenic disorders having in common dissolution of myofibrils and accumulation of inclusions, containing desmin and other myofibrillar and ectopic proteins. ${ }^{1-3}$ MFM manifesting clinically as skeletal and 
cardiac myopathy has been associated with mutations in several protein components of the $\mathrm{Z}$ disc. ${ }^{4,5}$ Recently, a c.8130G $>$ A (p.W2710X) mutation in the 24th immunoglobulin (Ig)-like repeat of filamin $\mathrm{C}$ has been identified in several German families as the cause of a novel type of predominantly limb-girdle myopathy (weakness most pronounced in proximal muscles of the lower limbs) having morphological features of MFM. ${ }^{6,7}$ Massive filamin C immunopositive aggregates were found in decaying myofibrils. Functional studies revealed that the mutant filamin $\mathrm{C}$ is incapable of forming dimers with wild-type filamin C. ${ }^{8}$

FLNC gene comprising $\sim 28.8 \mathrm{~kb}$ of genomic DNA and coding for 2725 amino acids is located within the 7q32q35 chromosomal band and is predominantly expressed in skeletal and cardiac muscles. ${ }^{9}$ The N-terminal portion of filamin $\mathrm{C}$ contains an actin-binding domain composed of two calponin homology domains (CHD1 and CHD2); this structure is followed by a semiflexible rod comprising 24 highly homologous tandem repeats, each containing 93 to 103 amino-acid residues and characterized structurally as Ig-like folds serving as interface for interaction with numerous filamin-binding proteins. ${ }^{9-11}$

Three highly related filamin proteins, FLNA, FLNB, and FLNC, interact with about 40 cellular proteins of great functional diversity. ${ }^{11}$ The best known filamin function is supporting intracellular filamentous networks through cross-linking actin. Filamin $\mathrm{C}$ cross-links actin at the Z-disc level and binds to Z-disc proteins, FATZ and myotilin, ${ }^{12-14}$ among others. In addition, filamin $C$ interacts with sarcoglycan- $\gamma$ and $-\delta$, components of the dystrophin-dystroglycan complex at the sarcolemma ${ }^{15}$ and acts as scaffold for transmembrane receptors, signaling and adapter proteins. ${ }^{11,16,17} \mathrm{~A}$ recent study based on analysis of FLNC-deficient mice indicates a role for filamin $\mathrm{C}$ in the muscle development and maintenance of muscle structural integrity. ${ }^{18}$ Mutations in filamins $\mathrm{A}$ and $\mathrm{B}$ have been reported to cause a broad range of congenital malformations affecting brain, bone, and other organs. ${ }^{17}$

The goal of this study was to assess the role of the FLNC gene mutations as a cause of MFM in a series of cases of diverse origins studied and documented by an International MFM collaborative group.

\section{Patients and methods Patients}

We studied several groups of patients: (1) North and South American patients of mixed ethnicity clinically and pathologically evaluated at the National Institute of Neurological Disorders and Stroke in Bethesda, MD, USA; (2) Spanish patients studied at the Institut de Neuropatologia, IDIBELL-Hospital de Bellvitge, Hospitalet de Llobregat, Barcelona, Spain; (3) patients of mixed ethnicity studied at three centers in the UK: Institute of Human
Genetics, Newcastle upon Tyne; Department of Clinical Neurology, Radcliffe Infirmary, Oxford; University Hospitals of Leicester; (4) French patients studied at the Institut de Myologie, Groupe Hospitalier Pitié-Salpêtrière, Paris, France; (5) patients of German ancestry referred by the Department of Neuropathology, Mainz University Medical Center, Germany, and the Institute of Neuropathology, Charité-Universitätsmedizin, Berlin, Germany; (6) patients from Poland evaluated at the Medical Research Center, Warsaw, Poland. Each of the participating institutions is a highly specialized regional (or national) referral center for patients with neuromuscular disorders. Patients with suspected MFM were systematically identified, studied and documented for more than 10 years. In each patient, the following investigations were carried out: pedigree analysis; neurological examination, including muscle strength evaluation according to the Medical Research Council grading scale, serum CK level assessment; electrophysiological studies that included nerve conduction testing and concentric needle EMG, muscle biopsy in at least a single member of an affected family, and when indicated cardiologic examination with electrocardiography (EKG) and echocardiography, muscle CT or MRI scanning, and respiratory function tests. We studied a total of 186 patients from 127 families; of these, in 88 families the disease was associated with pathogenic mutations in DES (MIM no. 125660), CRYAB (MIM no. 123590), MYOT (MIM no. 604103), or ZASP (MIM no. 605906) gene. As the remaining 39 families (that included 46 affected individuals) did not show mutations in any of these four genes, sequencing of the FLNC coding regions was carried out in the index cases.

Myofibrillar myopathy diagnostic criteria for patient selection were adopted from previous studies. ${ }^{1-7,19,20}$ Briefly, history of slowly progressive muscle weakness of distal and proximal lower limb muscles, possible association with cardiomyopathy, characteristic histopathological alterations in trichromatically stained muscle sections consisting of amorphous, hyaline, or granular material; immunocytochemical analysis showing abnormal ectopic expression of desmin and many other proteins; electron microscopy demonstrating myofibrillar degeneration and disintegration of the sarcomeres. A combination of these findings points to the diagnosis of MFM.

Only two patients, mother and daughter, from a German family 00-74 that was part of the International cohort showed a mutation in the FLNC gene. The disease onset was at the age of 60 and 34 years, respectively. The mother complained of difficulty in climbing stairs and at initial evaluation had paresis of pelvic muscles. Four years later, proximal upper extremity muscles became involved, at which time she was diagnosed with limb-girdle muscular dystrophy. At evaluation during the 12th year of illness, she had paresis of neck flexion and extension (4/5), reduced strength in deltoids (right $3 / 5$, left $4 / 5$ ), biceps, 
triceps brachii and brachioradialis (4/5), pelvic muscles $(1 / 5)$, knee flexors (2/5), extensors (4/5), and distal paresis of legs mainly involving the anterior compartment $(3-4 / 5)$. There was bilateral winging of the scapula. Paraspinal and abdominal muscles were also clearly involved. Standing and walking on heels or toes was not possible; the patient is using a walking frame. Tendon reflexes were diminished in upper and lost in the lower extremities. Electromyography showed signs of myopathy. Cardiac or respiratory muscles were not affected.

The daughter complained of muscle pain increasing with exercise and difficulty in climbing stairs. Two years into the illness, she was diagnosed with symmetric limb-girdle paresis (upper limbs 4/5; lower limbs 3-4/5) and hypotrophy of proximal upper-limb muscles. At evaluation in the 9 th year of illness, she had paresis of deltoids $(4 / 5)$, biceps and brachioradialis $(4 / 5)$, pelvic muscles $(1-2 / 5)$, knee flexors (4/5) and knee extensors (4/5), and distal leg muscle weakness $(3-4 / 5)$. There was bilateral winging of the scapula. Paraspinal and abdominal muscles were also weak. Tendon reflexes were diminished in the upper and lost in the lower extremities. Standing up from sitting position was next to impossible. Deep tendon reflexes were hypoactive and sensation intact. Disease progression was slow; no sign of cardiac or respiratory muscle involvement were present. Peripheral neuropathy was not evident in either patient clinically or elctrophysiologically. CK levels were moderately elevated. The maternal grandmother, maternal uncle, and a brother had slowly progressive muscle weakness, by history.

On the basis of clinical, myopathological data, and pedigree analysis both patients were diagnosed with hereditary MFM. After obtaining informed consent, blood was drawn and skeletal muscle biopsy was performed on both patients. Genetic studies were approved by the Institutional Review Board of the National Institute of Neurological Disorders and Stroke, NIH.

\section{Mutation detection and screening}

The affected mother and daughter, along with other patients diagnosed with MFM, were consecutively screened for the presence of mutations in DES, CRYAB, MYOT, ZASP, and FLNC genes known to be implicated in the causation of MFM. Genomic DNA was used as template for PCR amplification with primers constructed for each coding exon. Primer sequences are available per request. Amplification was carried out using optimal procedures designed for each segment. PCR-generated fragments were purified by using the QIAquick PCR Purification Kit (Qiagen, Valencia, CA, USA) and directly sequenced using DyeTerminator $^{\mathrm{TM}}$ sequencing protocol on an ABI Prism ${ }^{\circledR} 3100$ Genetic Analyzer (Applied Biosystems, Foster city, CA, USA). For precise identification of an apparent insertion/deletion, a 353-nucleotide FLNC exon-18intron-18-exon-19 PCR fragment was amplified from genomic DNA of each patient and subcloned using the TOPO TA Cloning Kit (Invitrogen, Carlbad, CA, USA). DNA extracted from 15 clones per patient was screened for insert size, and 4 selected clones from each patient were sequenced in both directions. Screening of 241 control individuals of European ancestry was accomplished by GeneScan methodology: fragment sizes were determined by electrophoresis on ABI Prism 3100 Genetic Analyzer and characterized with the use of GeneScan Analysis software v. 3.7 (Applied Biosystems).

Total mRNA was extracted from muscle biopsy tissue obtained from the affected daughter and a control individual using the RNeasy ${ }^{\mathrm{TM}}$ kit (Qiagen, Valencia, CA, USA). One microgram of total RNA was used for reverse transcription (RT) performed according to the qScript cDNA SuperMix protocol (Quanta BioSciences, Gaithersburg, MD, USA). Full-length cDNA was amplified with primers $\mathrm{F}$ (5'-GTGCGGGACTTTGAGATCAT-3') and R (5'-ATTCACCACAAAGGGGCTCT- ${ }^{\prime}$ ) and the transcripts were analyzed using GeneScan methodology as described above.

\section{Immunoblot analysis}

For analysis of skeletal muscle proteins, extract from biopsy tissue was electrophoresed in 6\% SDS-PAGE and transferred onto nitrocellulose membrane in a Semi-Dry Transfer System (BioRad, Madrid, Spain). Nonspecific binding was blocked and membrane was incubated with monoclonal mouse anti-filamin C antibody RR90 ${ }^{13}$ at 1:100 dilution, then washed and incubated with the corresponding secondary antibody labeled with horseradish peroxidase (Dako, Barcelona, Spain). The protein band was detected by chemiluminescence ECL method (Amersham Biosciences, Little Chalfont, UK) . The myosin band of $205 \mathrm{kDa}$ stained with Coomassie Brilliant Blue $\mathrm{R}$ (Sigma, St Louis, MO, USA) in the post-transfer gel was used as control of protein loading. For comparative purposes, a sample from a healthy control individual was similarly processed.

\section{Myopathological study}

Biopsy muscle tissue obtained from both patients of the German family was immediately frozen in liquid nitrogenchilled isopentane and processed by routine histological and histochemical techniques. Immunocytochemical analysis for desmin and ubiquitin (Dako; dilutions for desmin 1:20 and ubiquitin 1:100); $\alpha \mathrm{B}$-crystallin, myotilin, dystrophin (dys 1), dysferlin, and MHC-class I (Novocastra, Newcastle upon Tyne, UK; dilutions 1:500; 1:150; 1:20; 1:10; and 1:50, respectively); caveolin-3 (Transduction Labs, Franklin Lakes, NJ, USA; dilution 1:10), was performed using the streptavidin-biotin Super Sensitive ${ }^{\mathrm{TM}}$ IHC detection system (BioGenex, San Ramon, CA, USA). Filamin $\mathrm{C}$ immunohistochemistry was performed using a monoclonal anti-filamin $C$ antibody $\mathrm{RR}^{13}$ at dilution 1:25. Amyloid-like deposits were visualized using Congo 
Red staining and rhodamine optics. For ultrastructural analysis, a small sample of biopsy tissue was fixed in $2 \%$ glutaraldehyde, postfixed with $1 \%$ osmium tetroxide, and embedded in araldite. Ultrathin sections were stained with uranyl acetate and lead citrate.

\section{Results}

Mutation analysis of candidate genes and identification of filamin $\mathrm{C}$ deletion

Each proband or sporadic patient was consecutively tested for mutations in DES, CRYAB, MYOT, and ZASP genes by direct sequencing of coding exons. Of 127 probands, mutations were reliably identified in 88 (69\%), and 39 (31\%) did not show mutations in any of these genes (detailed report in preparation). The 39 probands not showing mutations in the listed four MFM-causing genes were screened for FLNC mutations. A family from Germany represented by a mother and her daughter was the only one showing a mutation in the FLNC gene. Analysis of nucleotide sequence of the FLNC exon 18 in cloned material led to the identification of a 12-bp deletion from nucleotide 2997 to 3008 (Figure 1a; nucleotide numbering adopted from NCBI no. NM_001458.4). The mutation is predicted to result in an in-frame four-residue deletion abolishing the p.930-VKYT-933 sequence. Subcloning and sequencing of the RT-PCR fragment of exon 18 amplified from the mRNA extracted from the affected daughter's biopsied muscle confirmed the presence of the c.2997_3008del (Figure 1b). This FLNC defect was also identified in the DNA sample from the affected mother. Size-fragment analysis of FLNC exon 18 in 241 unrelated individuals of European ancestry failed to uncover defects in exon 18, suggesting that p.V930_T933del is the candidate mutation associated with MFM in this family (GenBank submission 'FLNC_18del' no. EU848496). The mutation is located in an evolutionarily conserved seventh Ig-like domain of the FLNC gene (Figure 1c). Splicing between exons 17, 18, and 19 was verified by testing the patient's cDNA and proven unchanged. Immunoblot

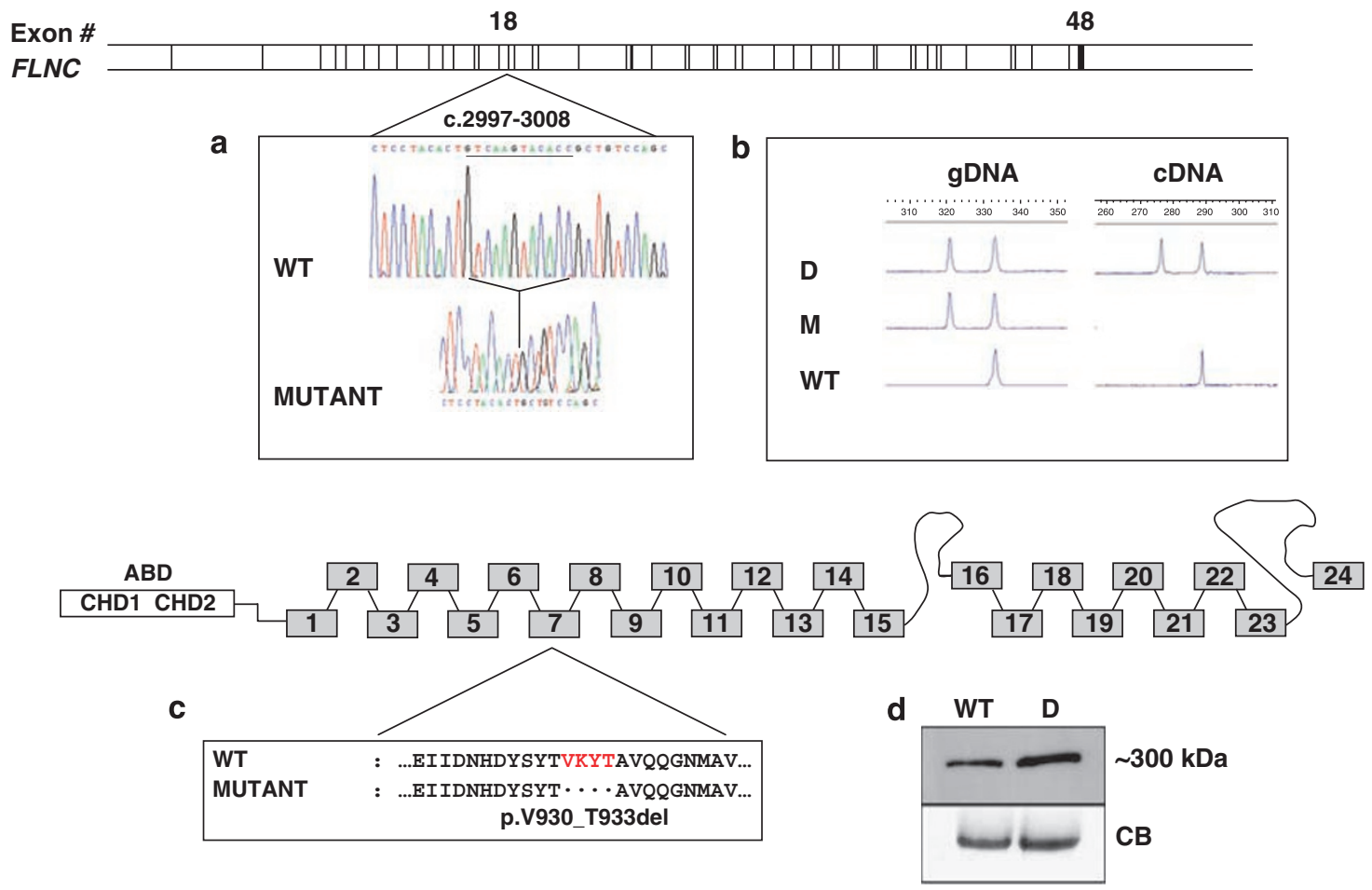

Figure 1 Molecular characterization of the identified FLNC mutation. Schematic drawing of the FLNC gene and filamin C protein (bottom part) showing the molecular effects of the FLNC deletion. (a) CDNA sequence alignment of a fragment of FLNC exon 18; the underlined 12 nucleotides (c.2997-3008) are deleted from the patient's sequence. (b) GeneScan profiles show a 12-bp difference in PCR-produced fragments amplified from the affected daughter's (D) and her mother's (M) genomic DNA (gDNA), compared with a single peak in the control (WT). Two transcript species with a 12-bp size difference were also identified in the biopsy muscle tissue obtained from the affected daughter, but only the longer transcript was present in the control sample. (c) The 12-nucleotide deletion in exon 18 is predicted to result in an in-frame deletion of four amino acids VKYT (p.Val930 Thr933del) within the seventh repeat of filamin C. (d) Immunoblot analysis of the muscle tissue proteins obtained from the patient (D) revealed a slight increase in the quantity of filamin C when compared with the control (WT). Myosin band of $205 \mathrm{kDa}$ stained with Coomassie Brilliant Blue $R(C B)$ shows equal protein loading. ABD, actin-binding domain; CHD 1 and 2, calponin homology domains. 
analysis performed on the index patient's muscle revealed slight increase in the quantity of filamin $\mathrm{C}$ when compared with the control (Figure 1d).

\section{Myopathological findings}

In the muscle biopsy obtained from the daughter, there was a marked variation in the fiber size with some atrophic fibers of both histochemical types, intermediate-size fibers as well as some hypertrophic fibers, also of both types. A few fibers contained internal nuclei. There was mild endomysial fibrosis (Figure 2). Muscle fiber necrosis, regeneration, or inflammatory infiltrates were not observed. Rimmed and unrimmed vacuoles were rarely seen. On trichrome-stained sections, several fibers contained abnormal areas featuring polymorphous hyaline and non-hyaline myofibrillary inclusions, which appeared dark-green to purple (Figure 2a, d and e). The inclusions varied in size, shape, and thickness; they were either single or multiple, or occupied the totality of the fiber. Very frequently, they had a convoluted serpentine appearance (Figure 2d, g and h). Furthermore, single or multiple spheroid bodies were seen in some fibers. Collections of small rod bodies were also observed. Some myofibrillar inclusions showed strong congophilia (Figure 2g). Acid phosphatase activity and PAS positivity were additional findings in some fibers. Oxidative activity was reduced in the fiber areas occupied by the inclusions but increased in the cytoplasm outside of inclusions. Single or multiple well-demarcated areas devoid of oxidative activity and resembling cores were observed in the cytoplasm of less damaged fibers (Figure 2c). ATPase activity was reduced in abnormal fiber regions (Figure $2 \mathrm{~b}$ ). There was type 1 fiber predominance. Immunohistochemical analysis showed prominent filamin $\mathrm{C}$, ubiquitin, desmin, myotilin, dystrophin (Figure $2 \mathrm{~h}-\mathrm{l}$ ), $\alpha \mathrm{B}$-crystallin, dysferlin, and caveolin-3 immunoreactivity in the abnormal fiber areas; no expression of MHC class 1 was observed in the sarcolemma of muscle cells.

Ultrastructural examination revealed major myofibrillar abnormalities, including accumulation of debris of Z-disc origin, granulofilamentous material, and the presence of a large number of nemaline rods. In addition, there were tubulofilamentous profiles and large autophagic vacuoles containing myelin-like figures and cellular debris. Mitochondrial aggregates were observed in the abnormal fiber regions (Figure $3 \mathrm{a}$ and $\mathrm{b}$ ).

Muscle biopsy samples obtained from the mother on the 2nd and 3rd years of her illness also showed increasingly clear signs of myopathy with many atrophic fibers and rimmed vacuoles.

\section{Discussion}

Myofibrillar myopathy is known to be associated with mutations in DES, CRYAB, MYOT, ZASP, and FLNC genes.
Differentiation between the gene-associated MFM subtypes on the basis of clinical/pathological phenotype alone is difficult. A precise diagnosis requires identification of a causative mutation. Of 127 families/sporadic patients with clinically/pathologically confirmed MFM, our study has identified only a single family showing the presence of FLNC mutation (less than 1\%), indicating that filaminopathy is a very rare subtype of MFM. The recently reported FLNC p.W2710X mutation in the 24th Ig-like repeat of filamin $\mathrm{C}$ was recognized as a cause of a distinct type of MFM in German families; the reported high frequency of this mutation in German patients is clearly dependent on a founder effect. ${ }^{7}$ The p.W2710X mutation was not found in MFM probands from the International cohort, four of them of German heritage. The family described in this communication had a four-residue deletion located within the seventh repeat of filamin C, the second known FLNC mutation causing an MFM syndrome.

Although this mutation is of a different type and located in a different functional domain of filamin $\mathrm{C}$, the phenotypic features of filaminopathy are similar to those observed in p.W2710X families (Supplementary Table S1). The disease is characterized by adult onset muscle weakness initially involving proximal muscles of lower limbs and spreading to upper limbs and distal muscles of lower extremities; paraspinal and abdominal muscle involvement and winging of the scapula were described in some p.W2710X cases and present in the current family, indicating that the distribution of muscle weakness is almost identical in all filaminopathy cases so far studied. Similarly to the p.W2710X cases, we observed myofibrillar inclusions strongly reacting with antibodies against filamin $\mathrm{C}$, desmin, myotilin, $\alpha \mathrm{B}$-crystallin, ubiquitin and other proteins, and frequent core-like and rod formations.

Disintegration of myofibrils and accumulation of large protein aggregates are the main features uniting filaminopathy with the other MFMs, but there are some slight distinctions that may be useful to consider for the differential diagnosis, determining priorities in genetic testing, and understanding disease mechanisms. Combining the information on myopathologic manifestations described in the p.W2710X families with our data, the myopathological features of filaminopathy can be summarized as follows: (a) the presence of rods appears to be a feature characteristic of filaminopathy; (b) filamentous profiles originally described in the p.W2710X family and also observed in the current family is another characteristic feature (these may also be seen in myotilinopathies ${ }^{21}$ ); (c) autophagic vacuoles are less common in filaminopathy than other MFM subtypes. ${ }^{21}$ Intracellular aggregates containing filamin C, desmin, myotilin and dystrophin, among other proteins, ${ }^{7}$ are not different from other MFM subtypes.

The mechanism by which the p.V930_T933del mutation leads to protein aggregation is currently unknown. 

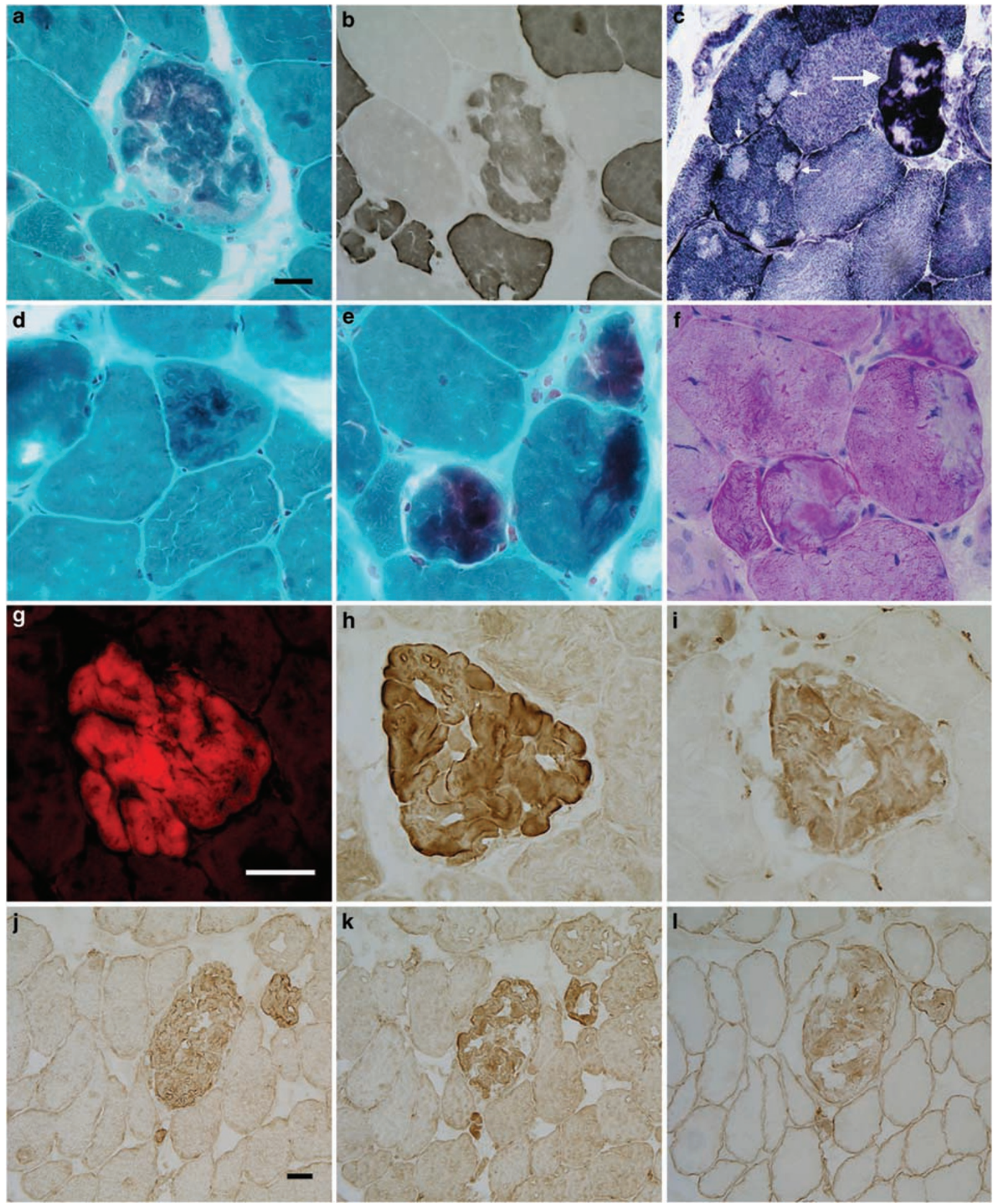

Figure 2 Histochemical and immunohistochemical findings in a patient with filaminopathy. (a) A large fiber containing a hyaline inclusion. (b) ATPase and (c) oxidative activities are partially reduced in the abnormal fiber area. Some adjacent fibers show several core-like lesions (arrows in c). Other types of lesions: (d) a thin, vermiform inclusion, (e) three muscle fibers containing demarcated abnormal areas, and (f) displaying some PAS positivity. Serial consecutive sections show an inclusion displaying congophilia (g), filamin C (h), ubiquitin (i), desmin (j), myotilin (k), and dystrophin (I) immunoreactivity. (a, d, and e) modified trichrome; (b) ATPase 4.35; (c) NADH; (f) PAS; (g) Congo Red; (h) filamin C; (i) ubiquitin; (j) desmin; (k) myotilin; and (I) dystrophin staining. Bar in $\mathbf{a}=40 \mu \mathrm{m}$ (refers to a through $\mathrm{f}$ ); bar in $\mathbf{g}=50 \mu \mathrm{m}$ (refers to $\mathrm{g}$ through $\mathbf{i}$ ); bar in $\mathbf{j}=25 \mu \mathrm{m}$ (refers to $\mathbf{j}$ through I). 

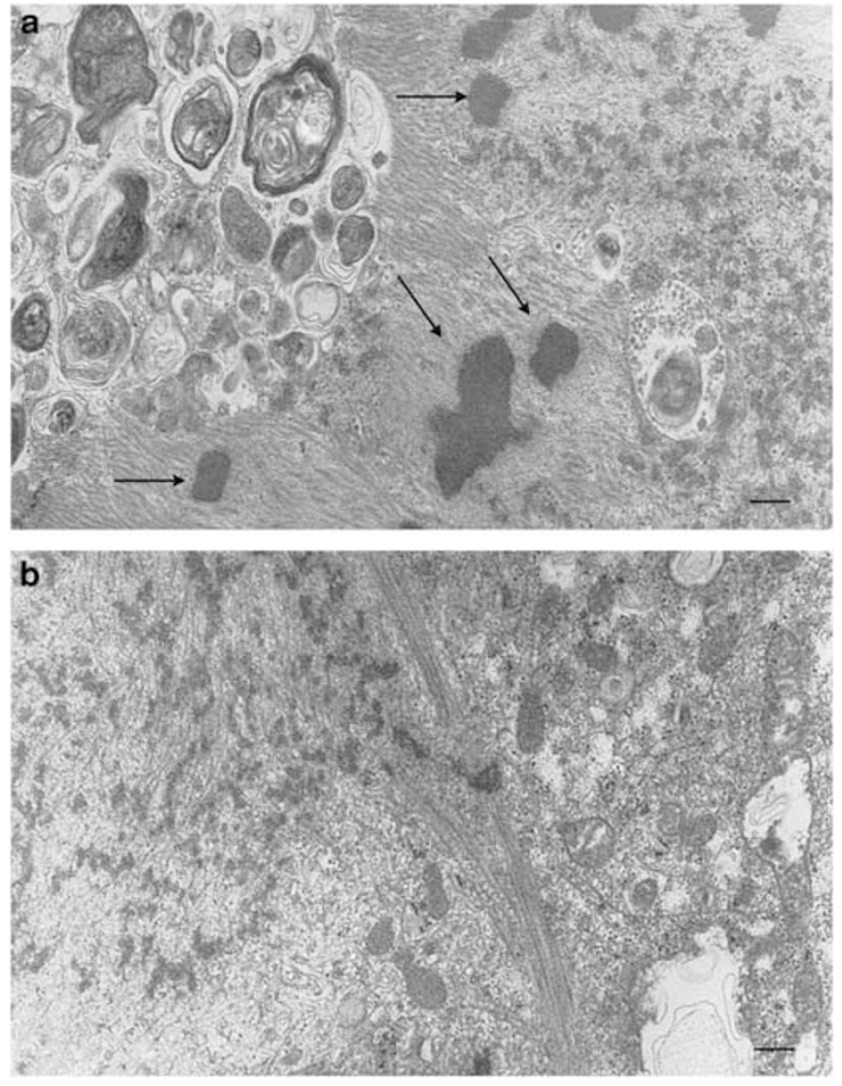

Figure 3 Ultrastructural analysis of skeletal muscle. (a) Widespread myofibrillary abnormalities, abundant rod formation (arrows), granulofilamentous material, and filamentous profiles. A large autophagic vacuole containing myeloid structures and membranous debris. (b) Remnants of filaments, Z-disc material, and foci of mitochondria. $\operatorname{Bar}=0.2 \mu \mathrm{m}$

Biochemical studies performed with the p.W2710X mutant have shown a disturbance in the secondary structure of the dimerization domain of filamin C. As a consequence, the mutant protein does not dimerize properly and has a tendency for uncontrolled filamin C aggregation; this in turn facilitates the recruitment of various proteins into the aggregates. ${ }^{6,7}$ The pathomechanisms involved in the p.V930_T933del-associated filaminopathy deserves further investigations.

A general feature of the filamins is that the Ig-fold domains may unfold under mechanical stress and spontaneously refold after the stress is over. ${ }^{22,23}$ This ability allows the molecule to be stretched to several times the length of its native size. This mechanism is considered critical for protection of the cytoskeletal architecture from being damaged in response to external deformation and most likely is a structural basis for the intrinsic flexibility of the actin networks. During unfolding, the filamin molecule may temporarily unbind its linkers. ${ }^{22,23}$ In-frame deletions in various genes often cause dysfunction of the encoded protein and are rarely encountered as DNA polymorphisms. A severe filamin C dysfunction due to the loss of several critical residues is most likely to occur. A similar inframe deletion of three residues in repeat 15 of the filamin A gene (c.4904_4912del, p.R1635_V1637del) was reported to cause skeletal deformities in a four-generation family. ${ }^{24}$

Molecular analysis of the novel filamin C mutation adds to the characterization of the diverse forms of MFMs and permits a more precise molecular diagnosis. Routine testing of MFM patients for filamin C mutations should be recommended in cases myopathologically proven as MFM and showing limb-girdle distribution of weakness. Further studies would allow establishing population frequency of this type of MFM and developing a better understanding of the structural and functional damage inflicted by FLNC mutations.

\section{Acknowledgements}

We are grateful to the members of the affected family for participation in the study. This research was supported in part by the Intramural Research Program of the National Institute of Neurological Disorders and Stroke, National Institutes of Health. MO was the recipient of the FIS 05-1213 grant. Additionally, MO and IF were supported by the Centro de Investigación Biomédica en Red de Enfermedades Neurodegenerativas (CIBERNED), Spain. Diagnostic and advisory service for rare neuromuscular disorders in the Newcastle upon Tyne area was funded by the UK National Commissioning Group; Newcastle University is a partner in the TREAT-NMD EU Network of Excellence (EC, 6th FP, proposal no. 036825; www.treat-nmd.eu). BG and PV were recipients of AFM Grant no. 12986 and the ANR Grant no. 06MRAR-039-01. HHG is a member of the German MD-NET. Filamin $C$ antibody was kindly provided by DO Fürst.

\section{References}

1 Nakano S, Engel AG, Waclawik AJ et al:: Myofibrillar myopathy with abnormal foci of desmin positivity. 1. Light and electron microscopy analysis of 10 cases. J Neuropathol Exp Neurol 1996: 55: 549-562.

2 Engel AG: Myofibrillar myopathy. Ann Neurol 1999; 46: 681-683.

3 Selcen D, Ohno K, Engel AG: Myofibrillar myopathy: clinical, morphological and genetic studies in 63 patients. Brain 2004; 127: 439-451.

4 Vicart P, Caron A, Guicheney P et al: A missense mutation in the alphaB-crystallin chaperone gene causes a desmin-related myopathy. Nat Genet 1998; 20: 92-95.

5 Selcen D, Engel AG: Myofibrillar myopathy. GeneReviews, (http:// www.genereviews.org/) 2008.

6 Vorgerd M, van der Ven PFM, Bruchertseifer V et al: A mutation in the dimerization domain of filamin $\mathrm{C}$ causes a novel type of autosomal dominant myofibrillar myopathy. Am J Hum Genet 2005; 77: 297-304.

7 Kley RA, Hellenbroich Y, van der Ven PF et al: Clinical and morphological phenotype of the filamin myopathy: a study of 31 German patients. Brain 2007; 130: 3250-3264.

8 Löwe T, Kley RA, van der Ven PF et al: The pathomechanism of filaminopathy: altered biochemical properties explain the cellular phenotype of a protein aggregation myopathy. Hum Mol Genet 2007; 16: $1351-1358$. 
9 Xie Z, Xu W, Davie EW, Chung DW: Molecular cloning of human ABPL, an actin-binding protein homologue. Biochem Biophys Res Commun 1998; 215: 914-919.

10 van der Flier A, Sonnenberg A: Structural and functional aspects of filamins. Biochim Biophys Acta 2001; 1538: 99-117.

11 Popowicz GM, Schleicher M, Noegel AA, Holak TA: Filamins: promiscuous organizers of the cytoskeleton. Trends Biochem Sci 2006; 31: 411-419.

12 Faulkner G, Pallavicini A, Comelli A et al: FATZ, a filamin-, actinin-, and telethonin-binding protein of the Z-disc of skeletal muscle. J Biol Chem 2000; 275: 41234-41242.

13 van der Ven PF, Obermann WM, Lemke B, Gautel $M$, Weber K, Fürst DO: Characterization of muscle filamin isoforms suggests a possible role of gamma-filamin/ABP-L in sarcomeric Z-disc formation. Cell Motil Cytoskeleton 2000; 45: 149-162.

14 Gontier Y, Taivainen A, Fontao L et al: The Z-disc proteins myotilin and FATZ-1 interact with each other and are connected to the sarcolemma via muscle-specific filamins. J Cell Sci 2005; 118: $3739-3749$.

15 Thompson TG, Chan Y-M, Hack AA et al: Filamin 2 (FLN2): a muscle-specific sarcoglycan interacting protein. J Cell Biol 2000; 148: $115-126$

16 Stossel TP, Condeelis J, Cooley L et al: Filamins as integrators of cell mechanics and signalling. Nature Rev Mol Cell Biol 2001; 2: $138-145$.
17 Feng Y, Walsh CA: The many faces of filamin: a versatile molecular scaffold for cell motility and signaling. Nat Cell Biol 2004; 6: 1034-1038.

18 Dalkilic I, Schienda J, Thompson TG, Kunkel LM: Loss of filamin $\mathrm{C}$ (FLNC) results in severe defects in myogenesis and myotube structure. Mol Cell Biol 2006; 26: 6522-6534.

19 Dalakas MC, Park K-Y, Semino-Mora C et al: Desmin myopathy, a skeletal myopathy with cardiomyopathy caused by mutations in the desmin gene. $N$ Engl J Med 2000; 342: 770-780.

20 Fardeau M, Godet-Guillain J, Tome FM et al: Une nouvelle affection musculaire familiale, definie par l'accumulation intrasarco-plasmique d'un materiel granulo-filamentaire dense en microscopie electronique. Rev Neurol 1978; 134: 411-425.

21 Claeys KG, Fardeau M, Schröder R et al: Electron microscopy in myofibrillar myopathies reveals clues to the mutated gene. Neuromuscul Disord 2008; 18: 656-666.

22 Furuike S, Ito T, Yamazaki M: Mechanical unfolding of single filamin A (ABP-280) molecules detected by atomic force microscopy. FEBS Lett 2001; 498: 72-75.

23 Yamazaki M, Furuike S, Ito T: Mechanical response of single filamin A (ABP-280) molecules and its role in the actin cytoskeleton. J Muscle Res Cell Motil 2002; 23: 525-534.

24 Stefanova M, Meinecke P, Gal A, Bolz H: A novel 9 bp deletion in the filamin A gene causes an otopalatodigital-spectrum disorder with a variable, intermediate phenotype. Am J Med Genet A 2005; 132: $386-390$.

Supplementary Information accompanies the paper on European Journal of Human Genetics website (http://www.nature.com/ejhg) 